\title{
Yarrowia lipolytica
}

National Cancer Institute

\section{Source}

National Cancer Institute. Yarrowia lipolytica. NCI Thesaurus. Code C114257.

A dimorphic yeast in the phylum Ascomycota. This species is strictly aerobic, degrades

hydrophobic substrates, assimilates glucose, fructose and mannose, and utilizes ethanol

and glycerol as carbon sources. Y. lipolytica is a biocatalyst and used commercially in

biotransformation to catalyze hydrolysis, esterification, interesterification and

transesterification reactions. It is also used for the production of citric acid. 